\title{
Inhaltsverzeichnis
}

Abkürzungsverzeichnis $\ldots \ldots \ldots \ldots \ldots \ldots \ldots \ldots \ldots$ IX

Literaturverzeichnis ................. XII

\section{Erster Tell}

Die Grundlagen des Inländerbehandlungsgrundsatzes im internationalen Urheberrecht

Erstes Kapitel: Die Entwicklung des internationalen Urheberrechts vor Abschluß der multilateralen Konvention . . . . . . . . . . . . . . 1

$\S 1$. Der Urheberrechtsschutz unter besonderer Berücksichtigung der Stellung fremder Urheber bis zum Abschluß der zweiseitigen Urheberrechtsabkommen . . . . . . . . . . . . 1

a. Das Privilegienwesen ... . . . . . . . . . . . . 1

b. Die Anfänge der gesetzlichen Anerkennung des Urheberrechts

$\S 2$. Die Bemühungen um die Verbesserung des Schutzes fremder Urheber auf Grund bilateraler Abkommen . . . . . . . . . 4

a. Die Rechtslosigkeit fremder Urheber ... . . . . . . . 4

b. Das napoleonische Dekret vom 19. Juni $1811 \ldots \ldots$. . . . . 4

c. Die Urheberrechtsabkommen im Deutschen Bund (1827 bis 1829) . . . . . . . . . . . . . . . . . . . . 4

d. Die Urheberrechtsabkommen vor Abschluß der multilateralen Konvention (1840 bis 1883) . . . . . . . . . . . . 5

e. Die Rechtsstellung fremder Urheber im Vorfeld der Berner

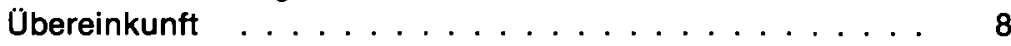

Zweites Kapitel: Der Ausbau des internationalen Urheberrechtsschutzes durch mehrseitige Konventionen $\ldots \ldots \ldots \ldots$

$\S 3$. Die Bildung und Weiterentwicklung der Berner Übereinkunft zum Schutze von Werken der Literatur und der Kunst vom 9. September 1886

a. Die Vorarbeiten (Festlegung der Schutzprinzipien)

b. Die ursprüngliche Fassung der Berner Übereinkunft und ihre Grundprinzipien . . . . . . . . . . . . . . . . .

c. Der Ausbau des Berner Verbands (Die Ergänzung von 1886 sowie die Revisionen von 1908, 1928, 1948, 1967 und 1971) $\quad \ldots \quad 19$ aa. Die Ergänzung von Paris (1896) _ . . . . . . . . . . . 20 
VIII

bb. Die Revision von Berlin (1908) _ . . . . . . . . . . . 21

cc. Die Revision von Rom (1928) . . . . . . . . . . . . . . . . 23

dd. Die Revision von Brüssel (1948) . . . . . . . . . . . . . 23

ee. Die Revision von Stockholm und Paris (1967/1971) . . . . 23

\$4. Die amerikanischen Urheberrechtsabkommen und ihre Schutzprinzipien (Überblick) . . . . . . . . . . . . . . . . . 26

a. Die Ausgangslage . . . . . . . . . . . . . . . 26

b. Die Konvention von Montevideo (1889) . . . . . . . . . . 27

c. Weitere bedeutsame amerikanische Urheberrechtsabkommen

§5. Die Bildung und Fortentwicklung des Welturheberrechtsabkommens vom 6. September 1952 . . . . . . . . . . . . . .

a. Der Anstoß zur Schaffung einer universellen Grundlage des internationalen Urheberrechtsschutzes . . . . . . . . . 29

b. Die Vorarbeiten . . . . . . . . . . . . . . . . . . 30

c. Die Schutzprinzipien der ursprünglichen Fassung . . . . . . 33

d. Die Pariser Revision des Welturheberrechtsabkommens (1971)

§6. Die bilateralen Urheberrechtsregelungen (Überblick) . . . . . . 35

a. Die Bedeutung der bilateralen Abmachungen für den internationalen Urheberrechtsschutz . . . . . . . . . . . . .

b. Die für den Schutz im Verhältnis zur Bundesrepublik Deutschland maßgebenden zweiseitigen Vereinbarungen und ihre Schutzprinzipien ................ 36

aa. Der deutsch-ägyptische Vertrag von $1951 \ldots \ldots$. . . . 36

bb. Das deutsch-iranische Abkommen von 1930 . . . . . . . 37

cc. Der deutsch-kolumbianische Vertrag von $1959 \ldots \ldots$

dd. Die besonderen Abkommen mit Verbandsländern der Berner Übereinkunft

\section{Zweiter Teil}

Der im Berner Verband und durch das Welturheberrechtsabkommen auf der Grundlage der Inländerbehandlung gewährte Rechtsschutz

Drittes Kapitel: Anwendungsbereich und Geltung der Konventionen . .

§ 7. Der Anwendungsbereich der Konventionen (Die Anknüpfung des Schutzes) . . . . . . . . . . . . . . . . . . 39

a. Vorbemerkungen zur Anknüpfung . . . . . . . . . . 39

b. Die Regelung der Anknüpfungspunkte . . . . . . . . 40

aa. Das System der Berner Übereinkunft . . . . . . . . . . . . 40

bb. Das System des Welturheberrechtsabkommens . . . . . 44 
§8. Der Anwendungsbereich der Konventionen (Die räumliche Abgrenzung des Schutzes

a. Die Beschränkung des Konventionsschutzes auf fremde Werke

b. Die Regelung der Berner Übereinkunft - Der Begriff des Ursprungslandes im Rahmen der verschiedenen Konventionsfassungen

c. Die Regelung des Welturheberrechtsabkommens

§9. Der Anwendungsbereich der Konventionen (Die zeitliche Abgrenzung des Schutzes)

a. Die Frage des zeitlichen Geltungsbereiches . . . . . . . . . . 52

b. Das Konzept der Konventionen . . . . . . . . . . . . . . 52 aa. Die Berner Übereinkunft . . . . . . . . . . . . . . . . 52 bb. Das Welturheberrechtsabkommen . . . . . . . . . 53

$\S 10$. Das Verhältnis der verschiedenen Konventionsfassungen $\quad \ldots \quad 54$

a. Die Problemstellung . . . . . . . . . . . . . . 54

b. Die Geltung der verschiedenen Fassungen der Berner Übereinkunft im Verhältnis der Verbandsländer zueinander . . . . . 55

c. Das Verhältnis der beiden Welturheberrechtsabkommen von 1952 und $1971 \ldots \ldots \ldots$. . . . . . . . . . 59

§ 11. Das Rangverhältnis der Konventionen untereinander . . . . . . 60

a. Die Überschneidung der Geltungsbereiche . . . . . . . . 60

b. Die Regelung im Verhältnis der Berner Übereinkunft - Welturheberrechtsabkommen ................ 61

c. Das Verhältnis des Welturheberrechtsabkommens zu dritten Urheberrechtsabkommen ................64

Viertes Kapitel: Der durch die Konventionen gewährte Rechtsschutz - Inländerbehandlung und weitere Schutzprinzipien . . . . . . . . 66

§12. Das räumliche Schutzprinzip . . . . . . . . . . . . 66

a. Der Ausschluß des „Ursprungs-" bzw. „Heimatlandes" des Werkes vom Konventionsschutz . . . . . . . . . . . . 66

b. Die Ausnahmeregelung der RBÜ zum Schutz vor Diskriminierung fremder Urheber in einem konventionsangehörigen Ur-

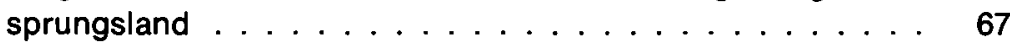

c. Der Schutz in den übrigen Konventionsländern . . . . . . . . 67

$\S 13$. Die materiellen Schutzprinzipien der Berner Übereinkunft und des Welturheberrechtsabkommens . . . . . . . . . . . . 67

a. Der Inländerbehandlungsgrundsatz . . . . . . . . 68

b. Die besonderen Konventionsrechte ... . . . . . . . 72

c. Die Berücksichtigung des Rechts des Ursprungslandes (Materielle Reziprozität) . . . . . . . . . . . . . . . . 72

d. Die Sonderregelung des Folgerechts in der RBÜ $\ldots \ldots \ldots 78$ 
$\mathbf{x}$

Fünftes Kapitel: Einzelprobleme der Inländerbehandlung . . . . . . . . 80

$\S 14$. Fragen des Umfangs der Verweisung . . . . . . . . . . . . . . . . 80

a. Die Regelung des Schutzgegenstandes . . . . . . . . . . . 80

b. Die Regelung der Befugnisse der geschützten Urheber . . . . 83

$\S 15$. Möglichkeiten der Einbeziehung neuer technischer Verwertungsmöglichkeiten und Verwertungsrechte . . . . . . . . . . . 85

a. Die Einbeziehung neuer technischer Verwertungsmöglichkeiten ......................... 85

b. Die Einbeziehung neuer Verwertungsrechte . . . . . . . . . . 87 aa. Die Einbeziehung des Folgerechts (Droit de Suite) . . . . . 87

bb. Die Einbeziehung der Büchereitantieme . . . . . . . . . 90

cc. Das Fotokopierproblem . . . . . . . . . . . 95 